Interfaces and Free Boundaries 11 (2009), 503-514

\title{
On the convexity of some free boundaries
}

\author{
ANTONIO GRECO \\ Dipartimento di Matematica e Informatica, Università di Cagliari, I-09124 Cagliari, Italy \\ E-mail: greco@unica.it \\ BERND KAWOHL \\ Mathematisches Institut, Universität zu Köln, D-50923 Köln, Germany \\ E-mail:kawohl@math.uni-koeln.de
}

[Received 5 May 2008 and in revised form 7 July 2009]

\begin{abstract}
We demonstrate that a method of Colesanti and Salani, which compares solutions of elliptic differential equations to their quasiconcave envelopes, can be extended to derive convexity of free boundaries. As examples we present the so-called dam problem, a free boundary problem modelling pollution and a Bernoulli problem. Moreover, we prove strict convexity of the wet region in the dam problem in arbitrary dimensions.
\end{abstract}

\section{Introduction}

In their paper [7] Colesanti and Salani study boundary value problems on convex rings, that is, domains $\Omega=\Omega_{0} \backslash \bar{\Omega}_{1}$ which are the difference of two open convex sets $\Omega_{i} \subset \mathbb{R}^{d}$ with $\Omega_{1} \subset \subset \Omega_{0}$. In the simplest case $\Delta u=0$ in $\Omega$, while $u=1$ on $\partial \Omega_{1}$ and $u=0$ on $\partial \Omega_{0}$ are imposed as boundary conditions, but other differential equations such as $\Delta_{p} u=f(u)$ with $f$ nondecreasing and $f(0)=0$ are also considered. If we set $u \equiv 1$ on $\bar{\Omega}_{1}$, then $u$ is well-defined everywhere in $\Omega_{0}$. It is known that solutions to such problems have convex level sets $\Omega_{t}=\left\{x \in \Omega_{0}: u(x) \geqslant t\right\}$ for $t \in(0,1)$.

One method of proof is the Gabriel-Lewis method that has been thoroughly studied in the 1980's in [13], [24], [19] and again more recently in [15]. It amounts to showing that $u((x+y) / 2) \geqslant$ $\min \{u(x), u(y)\}$ for every $(x, y) \in \Omega_{0}^{2}$.

Another method, which is conceptually very elegant, was developed by Colesanti and Salani in [7] and further developed in [10]. They define the quasiconcave envelope $u^{*}$ of $u$ as the smallest quasiconcave function above $u$. In other words, the level sets of $u^{*}$ are the convex hulls $\Omega_{t}^{*}$ of the level sets of $u$, and so by definition $u^{*} \geqslant u$. Then they derive a differential inequality for $u^{*}$. This inequality holds only in the sense of viscosity solutions, but it suffices to conclude, using an appropriate comparison principle, that $u^{*} \leqslant u$ in $\Omega$, because $u^{*}=u$ on $\partial \Omega$.

Incidentally, the method of comparing $u$ with a related function $u^{*}$ to show that $u=u^{*}$ has been successfully exploited in other geometrical contexts. In [2] and [18], $u^{*}$ is the convex envelope of $u$, i.e. the function whose graph is the convex hull of the graph of $u$, for which an interesting characterization has recently been given in [27]. In [30], $u^{*}$ represents the starshaped envelope of $u$.

In the present paper we shall study free boundary problems, in which $\Omega_{0}$ is chosen by the solution. Therefore its shape is not a priori known to be convex. In replacing $u$ by $u^{*}$ we replace a possibly nonconvex support of a function $u$ by its convex hull and thus modify a free boundary in order to show that it has to be convex from the beginning. Ingredients of the proof are the differential inequalities of Colesanti and Salani and a Hopf-type boundary lemma. 


\section{Preliminaries, differential inequalities for $u^{*}$}

In this section we consider a ring-shaped domain $\Omega$ as before, but we drop the assumption that $\Omega_{0}$ is convex (this is what we want to prove). We let $u$ be a function of class $C^{1}(\Omega) \cap C^{0}\left(\bar{\Omega}_{0}\right)$ satisfying $u=0$ on $\Gamma=\partial \Omega_{0}, u \equiv 1$ in $\bar{\Omega}_{1}$ and $0<u<1$ in $\Omega$. We suppose, further, that $u$ is of class $C^{2}$ in a neighborhood of every $x \in \Omega$ such that $\nabla u(x) \neq 0$. This is the case, for instance, if $u$ is a $p$-harmonic function, i.e., a solution of $\Delta_{p} u=0$ in $\Omega$ [31]. The results also apply to the case when $u$ is the solution to the dam problem discussed in Section 3 and $\Omega$ is its support (instead of being ring-shaped).

In the next sections we deal with solutions $u$ to some free boundary problems that can be compared with suitable one-dimensional supersolutions. To be more precise, let us consider a point $x \in \Omega$ and define $t=u(x)$. If the level set $\Omega_{t}$ has a supporting hyperplane $\pi$ passing through $x$, then we construct a supersolution $\bar{u}$ depending only on the distance from $\pi$ and attaining the value $t$ on $\pi$. Since $\nabla \bar{u} \neq 0$, we prove eventually that $\nabla u(x) \neq 0$. For the moment we take the following property as an assumption:

Nondegeneracy. If the level set $\Omega_{t}$, for $t$ satisfying $\inf _{\Omega} u<t<\sup _{\Omega} u$, has a supporting hyperplane passing through some $x \in \Omega_{t}$, then $\nabla u(x) \neq 0$.

Nondegeneracy implies the following minimum principle for $u^{*}$.

PROPOSITION 1 (A minimum principle) For every $t$ as above and for every $x$ interior to $\Omega_{t}^{*}$ we have $u^{*}(x)>t$.

Proof. We have to exclude that $u^{*}(x)=t$. The argument is by contradiction. Suppose that $u^{*}\left(x_{0}\right)=t$ at some $x_{0}$ interior to $\Omega_{t}^{*}$. Since $u^{*} \geqslant s$ in $\Omega_{s}^{*}$, we have $x_{0} \notin \Omega_{s}^{*}$ for $s>t$. The union $G_{t}=\bigcup_{s>t} \Omega_{s}^{*}$ is a convex set not containing $x_{0}$. The difference $\Omega_{t}^{*} \backslash G_{t}$ contains $x_{0}$, and has a nonempty interior because $x_{0}$ is interior to $\Omega_{t}^{*}$ and $G_{t}$ is convex. By construction we have $u^{*}(x)=t$ for all $x \in \Omega_{t}^{*} \backslash G_{t}$. Furthermore, there must be a point $P \in \Omega \backslash \bar{G}_{t}$ such that $u(P)=t$. Indeed, if $u(x)<t$ at every $x \in \Omega \backslash \bar{G}_{t}$ then $\Omega_{t}$ is a subset of the convex set $\bar{G}_{t}$, which implies $\Omega_{t}^{*} \subset \bar{G}_{t}$, but this has been excluded. Since $P$ is a local maximum point for $u$, we have $\nabla u(P)=0$.

Now if $\Omega$ is a ring-shaped domain as in the Introduction, we take $P$ as far as possible from a fixed point $Q \in \Omega_{1}$, and let $\pi$ be the hyperplane passing through $P$ and orthogonal to the segment $Q P$. Since $\pi$ supports $\Omega_{t}$ and $\nabla u(P)=0$, we reach a contradiction with nondegeneracy and the conclusion follows. If, instead, $\Omega$ and $u$ are as in the dam problem, then by looking for the boundary points where $u \geqslant t$ we see that $\bar{G}_{t}$ contains the convex hull of the four points $(0,0)$, $(a, 0),(0, H-\sqrt{2 t}),(0, h-\sqrt{2 t})$. Taking $P$ as far as possible from the segment connecting the last two points, we see that the line $\pi$ passing through $P$ and parallel to that segment supports $\Omega_{t}$, and by nondegeneracy the conclusion follows again.

We shall need the following useful representation of points $x \in \Omega^{*}$ (see [7, Proposition 3.1] for a similar result).

Proposition 2 (Representation) Take $x \in \Omega^{*}$ and define $t=u^{*}(x)$. If $\inf _{\Omega} u<t<\sup _{\Omega} u$ then $x$ is a convex combination of suitable $x_{1}, \ldots, x_{k} \in \Omega, k \leqslant d$, such that $u\left(x_{i}\right)=t$ and $\nabla u\left(x_{i}\right) \neq 0$ for $i=1, \ldots, k$. Furthermore, there exists a hyperplane $\pi$ supporting both $\Omega_{t}$ and $\Omega_{t}^{*}$ and containing every $x_{i}$. The gradients $\nabla u\left(x_{i}\right)$ are orthogonal to $\pi$ and point towards $\Omega_{t}^{*}$.

Proof. By Carathéodory's theorem [23, 28], the point $x$ is a convex combination of suitable $x_{1}, \ldots, x_{d+1}$ such that $u\left(x_{i}\right) \geqslant t$ for $i=1, \ldots, d+1$. Let us say that $x_{i}$ is relevant if the 
corresponding coefficient $\lambda_{i}$ is positive, and negligible if $\lambda_{i}=0$. By Proposition 11, we have $x \in \partial \Omega_{t}^{*}$. This implies that there exists a supporting hyperplane $\pi$ containing all the relevant $x_{i}$, say $x_{1}, \ldots, x_{k}$. In particular, we must have $k \leqslant d$. Since $\Omega_{t} \subset \Omega_{t}^{*}$, every relevant $x_{i}$ lies on $\partial \Omega_{t}$, hence $u\left(x_{i}\right)=t$. By nondegeneracy, the gradients $\nabla u\left(x_{i}\right)$ do not vanish, are orthogonal to $\pi$ and point towards $\Omega_{t}^{*}$.

Denoting by $n$ the normal to the hyperplane $\pi$ pointing towards $\Omega_{t}^{*}$, and letting $u_{n}=\partial u / \partial n$, let us finally prove a key result concerning the gradient and Hessian of $u^{*}$ in the viscosity sense. By a test function $\varphi$ touching $u^{*}$ from above at $x$ we mean, as usual, a function $\varphi$ of class $C^{2}$ in a neighborhood of $x$ such that $u^{*}-\varphi$ has a local maximum at $x$ and $u^{*}(x)=\varphi(x)$. The last condition justifies the terminology but is not essential.

PROPOSITION 3 Take $x \in \Omega^{*}$ and define $t=u^{*}(x)$. If $\inf _{\Omega} u<t<\sup _{\Omega} u$ then for every unit vector $v$ such that $v \cdot n \neq 0$, and for every sufficiently small $r$, the following estimate holds:

$$
u^{*}(x+r v) \geqslant u^{*}(x)+r v \cdot n\left(\sum_{j=1}^{k} \lambda_{j} / u_{n}\left(x_{j}\right)\right)^{-1}+o(r) .
$$

Furthermore, any test function $\varphi$ touching $u^{*}$ from above at $x$ satisfies

$$
\begin{gathered}
\nabla \varphi(x)=\left(\sum_{j=1}^{k} \lambda_{j} / u_{n}\left(x_{j}\right)\right)^{-1} n, \\
D^{2} \varphi(x) \geqslant\left(\sum_{j=1}^{k} \lambda_{j} / u_{n}\left(x_{j}\right)\right)^{-3} \sum_{j=1}^{k} \lambda_{j} D^{2} u\left(x_{j}\right) / u_{n}^{3}\left(x_{j}\right) .
\end{gathered}
$$

Inequalities similar to $[3$ are proved in [6, ineq. (8)] and in [25, ineq. (50)]. When we presented our results in Firenze on Dec. 12, 2008, Paolo Salani kindly provided us with a copy of [5] and pointed out that our Proposition 3 could also be derived from Prop. 2.2 in [5] in a way similar to the derivation of their Theorem 3.1. For the reader's convenience we now give our original proof.

Proof. By Proposition 2, the point $x$ is a convex combination $\sum_{i=1}^{k} \lambda_{i} x_{i}$ of suitable $x_{i} \in \Omega$ such that $u\left(x_{i}\right)=t$ for all $i$. Those points belong to a supporting hyperplane $\pi$ of $\Omega_{t}$ whose normal, directed towards $\Omega_{t}$, is denoted by $n$. Let us fix a unit vector $v \in \mathbb{R}^{d}$ satisfying $v \cdot n \neq 0$. By the implicit function theorem, there exist smooth functions $\rho_{i}(r), i=1, \ldots, k$, and $s(r)$, defined in a neighborhood of $r=0$, such that $\rho_{i}(0)=0$ for $i=1, \ldots, k, s(0)=t$ and

$$
\begin{gathered}
u\left(x_{i}+\rho_{i}(r) v\right)=s(r) \quad \text { for } i=1, \ldots, k, \\
\sum_{i=1}^{k} \lambda_{i} \rho_{i}(r)=r .
\end{gathered}
$$

Indeed, the Jacobian matrix of the preceding system at $\rho_{i}=0$ and $s=t$ is

$$
J=\left(\begin{array}{cccc}
u_{v}\left(x_{1}\right) & \ldots & 0 & -1 \\
\vdots & \ddots & \vdots & \vdots \\
0 & \ldots & u_{v}\left(x_{k}\right) & -1 \\
\lambda_{1} & \ldots & \lambda_{k} & 0
\end{array}\right)
$$

where $u_{v}=\partial u / \partial v$. Of course, the first $k$ rows of $J$ are linearly independent. Furthermore, the last row is independent from the preceding ones because in order to write it as their linear combination 
we must multiply the $i$-th row by $\lambda_{i} / u_{v}\left(x_{i}\right)$ and then sum over $i$. Looking at the last component we should obtain $\sum_{i} \lambda_{i} / u_{v}\left(x_{i}\right)=0$, but this is impossible because the sign of $u_{v}\left(x_{i}\right)$ is independent of $i$ and the coefficients $\lambda_{i}$ are positive. Hence the implicit function theorem applies, as claimed.

Observe that $u^{*}\left(x_{i}+\rho_{i}(r) v\right) \geqslant u\left(x_{i}+\rho_{i}(r) v\right)=s(r)$ for each $i$. Since $x+r v=\sum_{i} \lambda_{i}\left(x_{i}+\right.$ $\left.\rho_{i}(r) v\right)$, and since $u^{*}$ is quasiconcave, we have $u^{*}(x+r v) \geqslant s(r)$. Hence any test function $\varphi$ touching $u^{*}$ from above at $x$ satisfies $\varphi(x+r v) \geqslant s(r)$. Let us compute $s^{\prime}(0)$ and $s^{\prime \prime}(0)$. By differentiating (4)-(5) we get

$$
\begin{gathered}
u_{v}\left(x_{i}+\rho_{i}(r) v\right) \rho_{i}^{\prime}(r)=s^{\prime}(r) \quad \text { for } i=1, \ldots, k, \\
\sum_{i=1}^{k} \lambda_{i} \rho_{i}^{\prime}(r)=1 .
\end{gathered}
$$

A further differentiation at $r=0$ yields

$$
\begin{gathered}
u_{v v}\left(x_{i}\right)\left(\rho_{i}^{\prime}(0)\right)^{2}+u_{v}\left(x_{i}\right) \rho_{i}^{\prime \prime}(0)=s^{\prime \prime}(0) \quad \text { for } i=1, \ldots, k \\
\sum_{i=1}^{k} \lambda_{i} \rho_{i}^{\prime \prime}(0)=0
\end{gathered}
$$

where $u_{v v}=\partial^{2} u / \partial v^{2}$. From (6)-77) we obtain, at $r=0$,

$$
\begin{aligned}
& s^{\prime}(0)=\left(\sum_{j=1}^{k} \lambda_{j} / u_{v}\left(x_{j}\right)\right)^{-1}, \\
& \rho_{i}^{\prime}(0)=\left(u_{v}\left(x_{i}\right) \sum_{j=1}^{k} \lambda_{j} / u_{v}\left(x_{j}\right)\right)^{-1} .
\end{aligned}
$$

Using the last expression to replace $\rho_{i}^{\prime}(0)$ in 8 we get

$$
\rho_{i}^{\prime \prime}(0)=\left(u_{v}\left(x_{i}\right)\right)^{-1} s^{\prime \prime}(0)-\frac{u_{v v}\left(x_{i}\right)}{\left(u_{v}\left(x_{i}\right)\right)^{3}}\left(\sum_{j=1}^{k} \lambda_{j} / u_{v}\left(x_{j}\right)\right)^{-2} .
$$

By plugging this into 9 , and since $u_{v}\left(x_{j}\right)=u_{n}\left(x_{j}\right) n \cdot v$, we finally arrive at

$$
s^{\prime \prime}(0)=\left(\sum_{j=1}^{k} \lambda_{j} / u_{n}\left(x_{j}\right)\right)^{-3} \sum_{i=1}^{k} \lambda_{i} u_{v v}\left(x_{i}\right) / u_{n}^{3}\left(x_{i}\right) .
$$

From $\sqrt{10}$ rewritten as $s^{\prime}(0)=\left(\sum_{j} \lambda_{j} / u_{n}\left(x_{j}\right)\right)^{-1} n \cdot v$ we obtain $\sqrt{1}$, and 2 follows. By $(12)$, instead, we get (3). The fact that $(10)$ and $(12)$ have been derived for $n \cdot v \neq 0$ makes no problem since $\varphi$ is regular by definition.

Let us point out some consequences of the preceding result that were found in [7] by means of a different argument. Inequality (3) implies 


$$
\begin{aligned}
\varphi_{n n}(x) & \geqslant\left(\sum_{j=1}^{d} \lambda_{j} / u_{n}\left(x_{j}\right)\right)^{-3} \sum_{j=1}^{d} \lambda_{j} u_{n n}\left(x_{j}\right) / u_{n}^{3}\left(x_{j}\right), \\
\Delta_{\pi} \varphi & \geqslant\left(\sum_{j=1}^{d} \lambda_{j} / u_{n}\left(x_{j}\right)\right)^{-3} \sum_{j=1}^{d} \lambda_{j} \Delta_{\pi} u\left(x_{j}\right) / u_{n}^{3}\left(x_{j}\right),
\end{aligned}
$$

where $\Delta_{\pi} \varphi=\Delta \varphi-\varphi_{n n}$. Furthermore, since the $p$-Laplace operator $\Delta_{p} u$ can be represented as $\Delta_{p} u=|\nabla u|^{p-2}\left[(p-1) u_{n n}+\Delta_{\pi} u\right]$ provided that $\nabla u \neq 0$, and since $u$ is assumed to be nondegenerate in the sense defined above, the quasiconcave rearrangement $u^{*}$ satisfies the following differential inequality in the viscosity sense:

$$
\Delta_{p} u^{*}(x) \geqslant\left(\sum_{j=1}^{d} \lambda_{j} / u_{n}\left(x_{j}\right)\right)^{-(p+1)} \sum_{j=1}^{d} \lambda_{j} \Delta_{p} u\left(x_{j}\right) /\left(u_{n}\left(x_{j}\right)\right)^{p+1} .
$$

Consequently, if $u$ is a nondegenerate, weak solution of $\Delta_{p} u=f(u)$ in $\Omega$ with $f \geqslant 0$ then

$$
\Delta_{p} u^{*}(x) \geqslant f\left(u^{*}(x)\right) \quad \text { in the sense of viscosity solutions. }
$$

Several comparison principles for viscosity solutions are found in the literature: see, for instance, [3, Theorem 1], [8, Theorem 3.3], [26, Theorem 1.1]. However, since we shall compare $u$ and $u^{*}$ in a subset $G \subset \Omega$ where $\nabla u \neq 0$, and $u$ satisfies $\Delta_{p} u=f(u)$ in the classical sense, a very specific result is enough to proceed. For completeness, we prove a suitable statement hereafter.

Proposition 4 (Comparison principle) Let $G$ be a bounded, open subset of $\mathbb{R}^{d}$, and let $u$ be a function of class $C^{2}(\bar{G})$ satisfying $\inf _{G}|\nabla u|>0$. Thus, the function $\Delta_{p} u(x)$ is defined in the classical sense in $\bar{G}$. If $v \in C^{0}(\bar{G})$ is a viscosity solution of $\Delta_{p} v \geqslant \Delta_{p} u$ in $G$, then

$$
\sup _{G}(v-u)=\max _{\partial G}(v-u) .
$$

Proof. Suppose, by contradiction, that the function $w=v-u$ attains an interior maximum $M>$ $\max _{\partial G} w$. Define $\phi=u-c e^{\gamma x_{1}}$, where $x_{1}$ stands for the first component of the variable $x$, and $c, \gamma$ are real numbers. For every $\gamma \in \mathbb{R}$ we choose a sufficiently small $c=c(\gamma)>0$ such that (i) the function $w(x)+c(\gamma) e^{\gamma x_{1}}=v-\phi$ attains its maximum at some $x(\gamma) \in G$; (ii) $\sup _{G}|\nabla u-\nabla \phi|<$ $\frac{1}{2} \inf _{G}|\nabla u|=: r$. In particular, $\nabla \phi(x) \notin B(0, r)$ for all $x, \gamma$. Since $\phi$ is a test function at the point $x(\gamma)$ we may write $a^{i j}(\nabla u(x(\gamma))) u_{i j}(x(\gamma)) \leqslant a^{i j}(\nabla \phi(x(\gamma))) \phi_{i j}(x(\gamma))$, where $a^{i j}(\xi)=$ $\delta^{i j}|\xi|^{p-2}+(p-2)|\xi|^{p-4} \xi_{i} \xi_{j}$ for $\xi \in \mathbb{R}^{d} \backslash\{0\}$ and the summation convention is used. By plugging the term $\phi_{i j}(x(\gamma))=u_{i j}(x(\gamma))-c(\gamma) \gamma^{2} e^{\gamma x_{1}(\gamma)} \delta^{i 1} \delta^{j 1}$ into the preceding inequality, we obtain

$$
\left[a^{i j}(\nabla u(x(\gamma)))-a^{i j}(\nabla \phi(x(\gamma)))\right] u_{i j}(x(\gamma))+c(\gamma) \gamma^{2} e^{\gamma x_{1}(\gamma)} a^{11}(\nabla \phi(x(\gamma))) \leqslant 0 .
$$

By the mean value theorem, we may write

$$
a^{i j}(\nabla u(x(\gamma)))-a^{i j}(\nabla \phi(x(\gamma)))=c(\gamma) \gamma a_{1}^{i j}(\xi(\gamma)) e^{\gamma x_{1}(\gamma)}
$$

where $a_{1}^{i j}=\partial a^{i j} / \partial \xi_{1}$ and $\xi(\gamma)$ lies on the segment joining $\nabla u$ to $\nabla \phi$ in $\mathbb{R}^{d}$. With this replacement, provided that $\gamma \neq 0$, we arrive at

$$
a_{1}^{i j}(\xi(\gamma)) u_{i j}(x(\gamma))+\gamma a^{11}(\nabla \phi(x(\gamma))) \leqslant 0 .
$$


Now let $\gamma \rightarrow+\infty$. By construction, $\xi(\gamma)$ and $\nabla \phi(x(\gamma))$ stay bounded and far from the origin. Since $u \in C^{2}(\bar{\Omega})$, the term $a_{1}^{i j}(\xi(\gamma)) u_{i j}(x(\gamma))$ is also bounded and the (positive) coefficient $a^{11}(\nabla \phi(x(\gamma)))$ remains far from zero. Hence, the left-hand side in 14 tends to $+\infty$. This contradiction shows that the comparison principle holds.

In order to compare $u$ and $u^{*}$ on the free boundary $\Gamma$ we use the following statement, which is also needed to prove the existence of the set $G$ of Proposition 4

Proposition 5 Define $w(x)=u^{*}(x)-u(x)$ for $x \in \bar{\Omega}$. The function $w$ cannot have a positive maximum $M$ at $x_{0} \in \Omega \cup \Gamma$ if $\nabla u\left(x_{0}\right)=0$.

Proof. Assume that a positive maximum $M$ is attained at such an $x_{0}$. Then $u^{*}\left(x_{0}\right)=u\left(x_{0}\right)+M>0$. Furthermore, since $u<1$ in $\Omega \cup \Gamma$, we also get $u^{*}\left(x_{0}\right)<1$ and Proposition 3 applies. By 11 we have $u^{*}\left(x_{0}+r n\right) \geqslant u^{*}\left(x_{0}\right)+\varepsilon r$ for a convenient $\varepsilon>0$ and for all sufficiently small $r>0$. Since $\nabla u\left(x_{0}\right)=0$, we also have $u\left(x_{0}+r n\right)=u\left(x_{0}\right)+o(r)$. By subtracting the last equality from the preceding inequality we obtain $w\left(x_{0}+r n\right) \geqslant M+\varepsilon r-o(r)$, a contradiction.

\section{The dam problem}

The flow of groundwater through a rectangular dam, which is impervious at the bottom, leads after the Baiocchi transformation to the following free boundary problem. Let $D=(0, a) \times(0, b)$ be a rectangular domain, the cross section of the dam, and let $H$ and $h$ denote the height of water left and right of the dam, $0<h<H<b$. We look for a function $u$ that satisfies Dirichlet conditions $u=g$ on $\partial D$ and the differential equation $\Delta u=1$ on its support $\Omega=\{x \in D: u(x)>0\}$. Physically, $\Omega$ represents the wet region in the dam. The datum $g$ is specified as follows: $g\left(x_{1}, 0\right)=$ $\frac{1}{2 a}\left[a H^{2}-x_{1}\left(H^{2}-h^{2}\right)\right]$ and $g\left(x_{1}, b\right)=0$ for $x_{1} \in[0, a] ; g\left(0, x_{2}\right)=\frac{1}{2}\left(H-x_{2}\right)\left(H-x_{2}\right)^{+}$and $g\left(a, x_{2}\right)=\frac{1}{2}\left(h-x_{2}\right)\left(h-x_{2}\right)^{+}$for $x_{2} \in(0, b)$. Another way to characterize the solution is the obstacle problem of minimizing

$$
J_{1}(v):=\int_{D}\left\{\frac{|\nabla v|^{2}}{2}+v\right\} \mathrm{d} x
$$

on $K_{1}:=\left\{v \in W^{1,2}(D): v \geqslant 0\right.$ in $D$ and $v=g$ on $\left.\partial D\right\}$. It is easy to see that this problem has a unique solution $u$ with the required properties. Moreover, $u_{x}$ and $u_{y}$ are negative in $\Omega$ and the free boundary $\partial \Omega \cap D$ can be represented as a smooth function of $x$.

THEOREM 6 The solution $u$ of the dam problem is quasiconcave. Therefore all of its level sets are convex.

For the proof we compare $u^{*}$, the quasiconcave envelope of $u$, with $u$ on $\Omega$. Since $u_{x}, u_{y}<0$ in $\Omega$, the nondegeneracy assumption of Section 2 is satisfied. By Proposition 3 we know that $\Delta u^{*} \geqslant$ $\Delta u$ in $\Omega$ in the sense of viscosity solutions, so that $u^{*}-u$ attains any positive maximum on $\partial \Omega$. However, by definition $u^{*}=u$ on $\partial D$, so that a positive maximum can only occur at a point $x_{0}$ on the free boundary $\Gamma=\partial \Omega \cap D$. But this is impossible by Proposition 5 , because $\nabla u=0$ on $\Gamma$. Hence $u^{*}>u$ cannot hold anywhere in $\Omega$. Since $u^{*} \geqslant u$, the equality follows.

Note that this method of proof can carry over to higher dimensions, and that it proves the convexity of all level sets, not just the support. In three dimensions one can think of the dam as a wall that encloses a pool.

In [12] and [9] one can find proofs that the support is even strictly convex in the sense that $\Gamma$ has positive curvature. However, the arguments there work only in two dimensions. The (less sharp) fact 
that the free boundary $\Gamma$ contains no segments can be easily proved as follows. Assume, contrary to the claim, that the free boundary $\Gamma$ contains a segment $S$. After a suitable change of coordinates we see that $S$ lies on $\left\{x_{1}=0\right\}$ and $\Omega \subset\left\{x_{1}>0\right\}$. The proof aims to show that the solution $u$ coincides with $v=x_{1}^{2} / 2$ in $\Omega$, which is impossible because the boundary condition on the impervious bottom of the dam is not satisfied. Indeed, $u$ should be linear along the bottom. However, since $h<H$, we see that $v$ is quadratic there. To prove that $u \equiv v$, consider the harmonic function $w=u-v$ in $\Omega$. Since $w=|\nabla w|=0$ on $S$, it follows that $w$ is identically zero [14, Problem 2.2] and the proof is complete.

\section{The pollution problem}

Imagine a chemical seeping through the wall of a full tank that occupies $\Omega_{1}$ into an ambient medium, where it is consumed by a reactant. Stationary problems of this nature can be modelled as variational problems: Minimize

$$
J_{2}(v):=\int_{\mathbb{R}^{d}}\left\{\frac{|\nabla v|^{p}}{p}+F(v)\right\} \mathrm{d} x
$$

on $K_{2}:=\left\{v \in W^{1, p}\left(\mathbb{R}^{d}\right): v \equiv 1\right.$ on $\left.\bar{\Omega}_{1}\right\}$. Here $F: \mathbb{R} \rightarrow \mathbb{R}_{0}^{+}$is a convex, nondecreasing function of class $C^{1, \alpha}$ with $F(s)=0$ for $s \leqslant 0$. The minimizing function $u$ represents the dimensionless concentration of the chemical. If

$$
\int_{0}^{1} F(t)^{-1 / p} \mathrm{~d} t<\infty
$$

then the absorption term in the Euler equation $-\Delta_{p} u=-f(u)$, where $f=F^{\prime}$, becomes so strong for small $u$ that a minimizer has finite support. Let us call this the pollution problem.

THEOREM 7 If $\Omega_{1}$ is convex then $u$ is quasiconcave. Therefore the support of $u$ and all of its level sets are convex.

This theorem was derived in [21] for $p=2$, but here we give a different proof that applies to any $p \in(1, \infty)$. The existence of a unique weak solution follows from the direct methods in the calculus of variations. By standard comparison results with a radially symmetric situation one can show that solutions have compact support and that $u(x) \in[0,1)$ outside $\Omega_{1}$. Moreover, since $f(u)$ is bounded, $u$ is of class $C^{1, \alpha}$ according to standard regularity results from [31] and [11], but then $f(u(x))$ is Hölder continuous everywhere outside $\Omega_{1}$. Only for $p=2$ does this imply $u \in C^{2, \beta}\left(\mathbb{R} \backslash \bar{\Omega}_{1}\right)$. In fact, for $p \neq 2$ the $p$-Laplacian operator degenerates at points where $\nabla u=0$, and we expect this to happen on the free boundary $\partial \Omega_{0}$, but not in $\Omega=\{x: u(x) \in(0,1)\}$. How can we prove that $|\nabla u|>0$ in $\Omega$ ?

That the level sets of $u$ are starshaped with respect to any point in $\Omega_{1}$ follows from [20] or [30]. Without loss of generality suppose that $0 \in \Omega_{1}$. Then we know that radial derivatives of $u$ are nonpositive, but not negative as desired. A way out of this difficulty is the observation that we do not need $\nabla u$ to be nonzero everywhere, but only at special points $x_{i}$ that span nontrivial (and convex at most $(d-1)$-dimensional) components of $\partial \Omega_{t}^{*} \backslash \partial \Omega_{t}$. This is where the inequalities of Proposition 3 are exploited, and this goal motivates the following step in the proof.

LEMma 8 (Nondegeneracy) Let $x_{0} \in \Omega$, and define $t=u\left(x_{0}\right)$. If there exists a hyperplane $\pi$ passing through $x_{0}$ and supporting $\Omega_{t}$, then $\nabla u\left(x_{0}\right)$ does not vanish, is orthogonal to $\pi$ and points towards $\Omega_{t}$. 
Proof. The claim follows from the weak comparison principle, after having constructed a suitable supersolution. Without loss of generality, we may assume $x_{0}=0, \pi=\left\{x_{d}=0\right\}$, and $\Omega_{t} \subset$ $\left\{x_{d} \geqslant 0\right\}$. Since $\Omega_{0}$ is bounded, we have $\Omega_{0} \subset\left\{x_{d}>\mu\right\}$ for some negative $\mu$. The function $\bar{u}\left(x_{1}, \ldots, x_{d}\right)=t\left(1-x_{d} / \mu\right)$ satisfies $\Delta_{p} \bar{u}=0$ in $\mathbb{R}^{d}, \bar{u}=t$ on $\pi, \bar{u} \geqslant 0$ on $\partial \Omega_{0}$. By the weak comparison principle, $f(u) \geqslant 0$ implies that $u \leqslant \bar{u}$ in $\Omega \cap\left\{x_{d}<0\right\}$, and the conclusion follows.

Now we are in a position to give a proof of Theorem 7 By definition $u^{*} \geqslant u$, and since $f$ is nondecreasing, by (13) we have $\Delta_{p} u^{*} \geqslant \Delta_{p} u$. Let us first show that no positive maximum $M$ of $w(x)=u^{*}(x)-u(x)$ over $\bar{\Omega}$ can occur on the free boundary $\partial \Omega_{0}$. It is well known that $u \in C^{1, \alpha}$ through the free boundary (see, for instance, [31]). Hence, $\nabla u=0$ on $\partial \Omega_{0}$ and we may apply Proposition 5 This prevents $w$ from having a positive maximum on $\partial \Omega_{0}$, and therefore any positive maximum of $w(x)=u^{*}(x)-u(x)$ over $\bar{\Omega}$ must occur at a point $x_{0} \in \Omega$. Now we can no longer argue as in the proof of Theorem 6 , because the differential equation for $u$ is nonlinear. Hence, we proceed as follows. If $M=\sup _{\Omega} w>0$, then we set $m:=\max _{\partial \Omega} w$ and observe that there exists $c \in(m, M)$ such that $\nabla u$ does not vanish in the closure $\bar{G}$ of the set $G=\{x \in \Omega: w(x)>c\}$, for otherwise we would have a sequence $c_{n} \nearrow M^{-}$and a sequence $x_{n} \in \Omega$ such that $w\left(x_{n}\right)>c_{n}$ and $\nabla u\left(x_{n}\right)=0$. Passing to the limit we would find $x_{0} \in \Omega$ such that $w\left(x_{0}\right)=M$ and $\nabla u\left(x_{0}\right)=0$, contrary to Proposition 5 Hence, a set $G$ having the stated properties does exist. Of course, the supremum of $w$ in $G$ equals $M$, and is attained in the interior and not on the boundary (where $w=c$ ). But this contradicts Proposition 4 where we take $v=u^{*}$. Hence $\Omega_{0}$ is convex and $u^{*} \equiv u$ in $\Omega$, i.e. $u$ is quasiconcave.

REMARK 9 Since $u=u^{*}$, a further application of 11 shows that $x \cdot \nabla u(x)<0$ in all of $\Omega$. This sharpens the conclusion in [20] and [30, Theorem 5.1], asserting (in case $u \in C^{1, \alpha}$ ) that $x \cdot \nabla u(x) \leqslant 0$ in $\Omega$.

\section{Bernoulli's problem}

Bernoulli's problem describes the outcome of a galvanization process. A body $\Omega_{1}$ in a galvanization bath is covered by another substance. The galvanized body occupies a domain $\Omega_{0}$. Mathematically this is modelled as follows: Minimize

$$
J_{3}(v):=\int_{\mathbb{R}^{d}}\left\{\frac{|\nabla v|^{p}}{p}+\lambda \chi_{\{v>0\}}\right\} \mathrm{d} x
$$

on $K_{2}:=\left\{v \in W^{1, p}\left(\mathbb{R}^{d}\right): v \equiv 1\right.$ on $\left.\bar{\Omega}_{1}\right\}$. Here $\lambda$ is a positive constant and $\chi_{A}$ the characteristic function of a set $A$.

THEOREM 10 The solution $w$ of Bernoulli's problem is quasiconcave. Therefore its support and all of its level sets are convex.

Several proofs of this result are known for various $p$ and $n$ (see e.g. [4], [22], [1], [16], [17]). The following proof is based on a strategy similar to the one used in Section 4 Define $\Omega=\Omega_{0} \backslash \bar{\Omega}_{1}$ and $\Omega_{t}=\{x \in \mathbb{R}: u(x) \geqslant t\}$, as before. Recall that if $\partial \Omega_{0}$ is sufficiently smooth, then $|\nabla u|$ attains the constant value $a=(p \lambda /(p-1))^{1 / p}$ there. Let us start with a key observation:

LEMMA 11 (Nondegeneracy) Let $x_{0}$ be a point of $\Omega$ such that $u\left(x_{0}\right) \in(0,1)$, and define $t=u\left(x_{0}\right)$. If there exists a hyperplane $\pi$ passing through $x_{0}$ and supporting $\Omega_{t}$, then $\left|\nabla u\left(x_{0}\right)\right|>a$. 
Proof. Let $n$ be the normal to $\pi$ directed towards $\Omega_{t}$. Consider the affine (and $p$-harmonic) function $z(x)=t+a\left(x-x_{0}\right) \cdot n\left(x_{0}\right)$. The following comparison argument shows that $\Omega_{0} \subset\left\{x \in \mathbb{R}^{d}\right.$ : $z(x)>0\}$. Denote by $u_{R, t}$ the (radial) minimizer of the same functional under the constraint $u_{R, t}=t$ in the closed ball $\bar{B}_{R}$. If $\Omega_{t} \subset \bar{B}_{R}$, then $u \leqslant u_{R, t}$ outside $\Omega_{t}$. Now let $R \rightarrow+\infty$ while the center of $B_{R}$ also goes to infinity, in such a way that $\partial B_{R} \rightarrow \pi$. Since the function $u_{R, t}$ is easily found by integration, we see that $u_{R, t} \rightarrow z$. Hence $\Omega_{0} \subset\left\{x \in \mathbb{R}^{d}: z(x)>0\right\}$, as claimed. Furthermore, $u \leqslant z$ in $D=\{x \in \Omega: 0<z(x)<t\}$ and $u\left(x_{0}\right)=z\left(x_{0}\right)$, which in turn implies $\left|\nabla u\left(x_{0}\right)\right| \geqslant a$. Consequently, $u$ is a classical solution of the nondegenerate elliptic quasilinear equation $\Delta_{p} u=0$ in a neighborhood of $x_{0}$. The difference $w=u-z$ satisfies therefore a linear elliptic inequality of the form $L w \geqslant 0$ near $x_{0}$ (see [14, Theorem 10.1]). Observe that the domain $D$ satisfies an interior sphere condition at $x_{0}$, hence we may apply Hopf's lemma and deduce the strict inequality $\left|\nabla u\left(x_{0}\right)\right|>a$.

Combining Proposition 2 and Lemma 11 we obtain:

LEMma 12 (Bound from below for $u^{*}$ ) Let $x_{0}$ be a point of $\Omega^{*}$ such that $u^{*}\left(x_{0}\right)=t \in(0,1)$. There exist positive numbers $\varepsilon, \rho$ and a unit vector $n$ such that the ball $\mathcal{B}=B\left(x_{0}+\rho n, \rho\right)$ centered at $x_{0}+\rho n$ and of radius $\rho$ is included in $\Omega_{t}^{*}$, and the restriction of $u^{*}$ to $\mathcal{B}$ is bounded from below as follows: $u^{*}(x)>t+(a+\varepsilon) n \cdot\left(x-x_{0}\right)$ for $x \in \mathcal{B}$.

Proof. Let us apply Proposition 2 at the point $x=x_{0}$. Let $\pi$ and $x_{i}, i=1, \ldots, k$, have the meaning given there, and let $n$ be the normal to $\pi$ pointing towards $\Omega_{t}^{*}$. By Lemma 11 there exists $\varepsilon>0$ so small that $a+\varepsilon<\left|\nabla u\left(x_{i}\right)\right|$ for all $i$. Denoting by $\eta$ a variable in $\mathbb{R}^{d}$, and by the implicit function theorem, for each $i$ there exists a neighborhood $U_{i}$ of the origin such that the function $\varphi_{i}(\eta)=u\left(x_{i}+\eta\right)-t-(a+\varepsilon) n \cdot \eta$ vanishes along a regular hypersurface $\Gamma_{i} \subset U_{i}$ dividing $U_{i}$ into two parts. Note that the hypersurfaces $\Gamma_{i}$ are orthogonal to $n$ at the origin. Since the index $i$ ranges in a finite set, there exists a sufficiently small radius $\rho>0$ such that the ball $B(\rho n, \rho)$ is contained in the set $U_{i} \cap\left\{\varphi_{i}(\eta)>0\right\}$ for every $i$. This may be rewritten as follows:

$$
u\left(x_{i}+\eta\right)>t+(a+\varepsilon) n \cdot \eta \quad \text { for } \eta \in B(\rho n, \rho) .
$$

Since $u^{*} \geqslant u$ by definition, we obtain $u^{*}\left(x_{i}+\eta\right)>t+(a+\varepsilon) n \cdot \eta$. Since $x_{0}+\eta$ is a convex combination of the points $x_{i}+\eta$, and since $u^{*}$ is quasiconcave, it follows that $u^{*}\left(x_{0}+\eta\right)>t+$ $(a+\varepsilon) n \cdot \eta$ in $B(\rho n, \rho)+\rho n$, which is equivalent to the claim.

As a consequence of Lemma 12, the difference $w=u^{*}-u$ cannot have a positive maximum at any $x_{0} \in \partial \Omega_{0}$ provided that $\left|\nabla u\left(x_{0}\right)\right|=a$ in the classical sense. The argument is the same as in the proof of Proposition 5 Since the last equality does not hold, in general, we proceed as follows.

Lemma 13 Define $w(x)=u^{*}(x)-u(x)$ for $x \in \bar{\Omega}$. The function $w$ cannot have a positive maximum at any $x_{0} \in \partial \Omega_{0}$.

Proof. Suppose, contrary to the claim, that $w$ attains a positive maximum at some $x_{0} \in \partial \Omega_{0}$. The first part of the proof aims to deduce from this assumption an estimate of $u$ near $x_{0}$, namely (3) below. Since $u\left(x_{0}\right)=0$ and $w\left(x_{0}\right)>0$, it follows that $u^{*}\left(x_{0}\right)>0$. Clearly $u^{*}\left(x_{0}\right)<1$ and therefore we may apply Lemma 12 Define $t=u^{*}\left(x_{0}\right)$ as in that lemma. Before proceeding further, note that the interior of $\Omega_{t}^{*}$ is included in $\Omega_{0}$. Indeed, by assumption we have

$$
u^{*}(x)-u(x) \leqslant t \quad \text { in } \Omega_{0},
$$


hence $u^{*}(x) \leqslant t$ on $\partial \Omega_{0}$; furthermore, by Proposition 1 we see that $u^{*}(x)>t$ for $x$ interior to $\Omega_{t}^{*}$. Hence the interior of $\Omega_{t}^{*}$ does not intersect $\partial \Omega_{0}$, but since it does intersect $\Omega_{0}$, it must be included in $\Omega_{0}$, as claimed. Consequently, the ball $\mathcal{B}=B\left(x_{0}+\rho n, \rho\right)$ of Lemma 12 is also included in $\Omega_{0}$, and by 2 , we may write

$$
u(x)>(a+\varepsilon) n \cdot\left(x-x_{0}\right) \text { in } \mathcal{B} .
$$

This inequality tells us, in a somewhat weak form, that $\left|\nabla u\left(x_{0}\right)\right|>a$, although $\nabla u\left(x_{0}\right)$ may not exist in the classical sense. In order to reach a contradiction, let us construct a radial minimizer $u_{R, s}$ having $\mathcal{B}$ as its support and attaining a suitable constant value $s$ in a concentric ball $B_{R}=$ $B\left(x_{0}+\rho n, R\right) \subset \mathcal{B}$ so that $u(x)>s$ in the closure $\bar{B}_{R}$. Of course, $u_{R, s}$ can be seen as a function of $r=\left|x-x_{0}-\rho n\right|$. By applying the divergence theorem to the equation $\Delta_{p} u_{R, s}=0$ it follows that $u_{R, s}(r)$ can be constructed as the solution to the first-order initial-value problem

$$
\begin{aligned}
r^{d-1}\left(-u_{R, s}^{\prime}(r)\right)^{p-1} & =\rho^{d-1} a^{p-1}, \quad r \in(R, \rho), \\
u_{R, s}(\rho) & =0,
\end{aligned}
$$

where $u_{R, s}^{\prime}=\mathrm{d} u_{R, s} / \mathrm{d} r$. Note that $u_{R, s}^{\prime}(\rho)=-a$. Moreover, the value of $s$ is related to $R$ by $s=u_{R . s}(R)$. By choosing $R$ close enough to $\rho$ (or equivalently by choosing $s$ small enough) and taking (3) into account, we get $u(x)>s$ in $\bar{B}_{R}$, as desired. Now we translate the function $u_{R, s}$ by some small $\sigma>0$ in the direction of $-n$, i.e., we pass from $u_{R, s}(x)$ to $v(x)=u_{R, s}(x+\sigma n)$. The support of $v$ is clearly $\mathcal{B}-\sigma n$, and the value $s$ is attained in the ball $B_{R}-\sigma n$. By choosing $\sigma$ small enough, we find that $u(x)>s$ on the last ball, and $x_{0}$ lies in the annulus $\left(\mathcal{B} \backslash \bar{B}_{R}\right)-\sigma n$. Let us see why this leads to a contradiction. Denote by $S$ the set of all $x \in \mathcal{B}-\sigma n$ such that $v(x)>u(x)$. The set $S$ is open, and nonempty because it contains $x_{0}$. The set $S_{0}=S \cap \Omega_{0}$ is also nonempty because the point $x_{0}$ is interior to $S$ and belongs to $\partial \Omega_{0}$. Since $u \equiv 0$ outside $\Omega_{0}$, we have $S_{0} \neq S$. We may decompose $J_{3}(v)$ as follows:

$$
J_{3}(v)=\int_{(\mathcal{B}-\sigma n) \backslash S}\left\{\frac{|\nabla v|^{p}}{p}+\lambda\right\} \mathrm{d} x+\int_{S}\left\{\frac{|\nabla v|^{p}}{p}+\lambda\right\} \mathrm{d} x .
$$

Now we compare the last integral with the one obtained by replacing the domain of integration $S$ with $S_{0}$ and the function $v$ with $u$. More precisely, we claim that

$$
\int_{S}\left\{\frac{|\nabla v|^{p}}{p}+\lambda\right\} \mathrm{d} x<\int_{S_{0}}\left\{\frac{|\nabla u|^{p}}{p}+\lambda\right\} \mathrm{d} x .
$$

To see this, define $\underline{u}(x)=\min \{u(x), v(x)\}$ and observe that

$$
J_{3}(\underline{u})=\int_{(\mathcal{B}-\sigma n) \backslash S}\left\{\frac{|\nabla v|^{p}}{p}+\lambda\right\} \mathrm{d} x+\int_{S_{0}}\left\{\frac{|\nabla u|^{p}}{p}+\lambda\right\} \mathrm{d} x .
$$

If inequality (5) were violated, we would have $J_{3}(\underline{u}) \leqslant J_{3}(v)$. Since $\underline{u} \equiv s$ on the ball $B_{R}-s n$, and since $J_{3}(v)$ is the minimum of $J_{3}$ under that constraint, we would arrive at $J_{3}(\underline{u})=$ $J_{3}(v)$, contradicting the fact that the minimizer under that constraint is unique and radial. Hence inequality (5) must hold, as claimed. To complete the proof, define $\bar{u}(x)=\max \{u(x), v(x)\}$. We have

$$
J_{3}(\bar{u})=\int_{\Omega_{0} \backslash S}\left\{\frac{|\nabla u|^{p}}{p}+\lambda\right\} \mathrm{d} x+\int_{S}\left\{\frac{|\nabla v|^{p}}{p}+\lambda\right\} \mathrm{d} x
$$


and also

$$
J_{3}(u)=\int_{\Omega_{0} \backslash S}\left\{\frac{|\nabla u|^{p}}{p}+\lambda\right\} \mathrm{d} x+\int_{S_{0}}\left\{\frac{|\nabla u|^{p}}{p}+\lambda\right\} \mathrm{d} x .
$$

By $\left[5\right.$, we get $J_{3}(\bar{u})<J_{3}(u)$, but this is impossible because $\bar{u} \equiv 1$ on $\Omega_{1}$, and $u$ minimizes $J_{3}$ under that constraint by assumption. The lemma follows.

Now we can give a proof of Theorem 10 Lemma 13 prevents the maximum in $\bar{\Omega}$ of the difference $w=u^{*}-u$ from being attained on $\partial \Omega_{0}$ unless $w \equiv 0$. Furthermore, if an interior, positive maximum $M$ is attained at some $x_{0} \in \Omega$, then Lemma 12 implies that

$$
\left|\nabla u\left(x_{0}\right)\right|>a,
$$

because the variation of $u$ must coincide with the one of $u^{*}$. Arguing as in the proof of Theorem 7 . and using (9) in place of Proposition 5, we still find a convenient neighborhood $G$ of $x_{0}$ such that $\nabla u \neq 0$ in $G$ and $w<M$ on $\partial G$. Since this contradicts Proposition 4, we must have $u^{*}=u$, as claimed.

\section{Acknowledgements}

This research was financially supported by Università di Cagliari. Its subject is part of the ESF Programme "Global and geometrical aspects of nonlinear partial differential equations (GLOBAL)".

\section{REFERENCES}

1. Acker, A., \& Meyer, R. A free boundary problem for the $p$-Laplacian: uniqueness, convexity and successive approximation of solutions. Electron. J. Differential Equations 1995, no. 8, 20 pp. Zbl 0820.35037 MR 1334863

2. Alvarez, O., Lasry, J.-M., \& Lions, P.-L. Convex viscosity solutions and state constraints. J. Math. Pures Appl. 76 (1997), 265-288. Zbl 0890.49013 MR 1441987

3. BARles, G., \& BUSCA, J. Existence and comparison results for fully nonlinear degenerate elliptic equations without zeroth-order term. Comm. Partial Differential Equations 26 (2001), 2323-2337. Zbl 0997.35023 MR 1876420

4. Beurling, A. On free-boundary problems for the Laplace equations. In: Sem. on Analytic Functions 1, Inst. Adv. Stud., Princeton (1957), 248-263. Zbl 0099.08302

5. Bianchini, C., Longinetti, M., \& SAlani, P. Quasiconcave solutions to elliptic problems in convex rings. Indiana Univ. Math. J. 58 (2009), 1565-1590. Zbl pre05603943

6. Cardaliaguet, P., \& Tahraoui, R. On the strict concavity of the harmonic radius in dimension $N \geqslant 3$. J. Math. Pures Appl. 81 (2002), 223-240. Zbl 1027.31003 MR 1894062

7. Colesanti, A., \& Salani, P. Quasi-concave envelope of a function and convexity of level sets of solutions to elliptic equations. Math. Nachr. 258 (2003), 3-15. Zbl 1128.35332 MR 2000041

8. CRAndall, M. G., Ishit, H., \& LiOns, P.-L. User's guide to viscosity solutions of second order partial differential equations. Bull. Amer. Math. Soc. 27 (1992), 1-67. Zbl $0755.35015 \mid$ MR 1118699

9. CRYER, C. W. A proof of the convexity of the free boundary for porous flow through a rectangular dam using the maximum principle. J. Inst. Math. Appl. 25 (1980), 111-120. Zbl 0436.76070 MR 0571973

10. CuOGhi, P., \& SALANi, P. Convexity of level sets for solutions to nonlinear elliptic problems in convex rings. Electron. J. Differential Equations 2006, no. 124, 12 pp. Zbl 1128.35320 MR 2255239 
11. DiBenedetto, E. $C^{1+\alpha}$ local regularity of weak solutions of degenerate elliptic equations. Nonlinear Anal. 7 (1983), 827-850. Zbl 0539.35027 MR 0709038

12. Friedman, A., \& Jensen, R. Convexity of the free boundary in the Stefan problem and in the dam problem. Arch. Ration. Mech. Anal. 67 (1977), 1-24. Zbl 0375.35028 MR 0473315

13. GABRiEL, R. M. A result concerning convex level surfaces of 3-dimensional harmonic functions. J. London Math. Soc. 32 (1957), 286-294. Zbl 0087.09702 MR 0090662

14. Gilbarg, D., \& Trudinger, N. S. Elliptic Differential Equations of Second Order. 2nd ed., Grundlehren Math. Wiss. 224, Springer, Heidelberg (1998).

15. Greco, A. Quasi-concavity for semilinear elliptic equations with non-monotone and anisotropic nonlinearities. Boundary Value Problems 2006, art. ID 80347, 15 pp. Zbl 1136.35363 MR 2211400

16. Gustafsson, B., \& Shahgholian, H. Existence and geometric properties of of solutions of a free boundary problem in potential theory. J. Reine Angew. Math. 473 (1996), 137-179. Zbl 0846.31005 MR 1390686

17. Henrot, A., \& Shahgholian, H. Existence of classical solutions to a free boundary problem for the $p$-Laplace operator: (I) the exterior convex case. J. Reine Angew. Math. 521 (2000), 85-97. Zbl 0955.35078 MR 1752296

18. Imbert, C. Convexity of solutions and $C^{1,1}$ estimates for fully nonlinear elliptic equations. J. Math. Pures Appl. 85 (2006), 791-807. Zbl pre05186555 MR 2236244

19. Kawohl, B. Rearrangements and Convexity of Level Sets in PDE. Lecture Notes in Math. 1150, Springer, Heidelberg (1985). Zbl 0593.35002 MR 0810619

20. Kawohl, B. On starshaped rearrangement and applications. Trans. Amer. Math. Soc. 296 (1986), 377386. Zbl 0612.35033 MR 0837818

21. Kawohl, B. On the convexity and symmetry of solutions to a free boundary problem. J. Austral. Math. Soc. Ser. A 42 (1987), 57-68. Zbl 0619.35051 MR 0862721

22. KAwOHL, B. On the convexity of level sets for elliptic and parabolic exterior boundary value problems. In: Potential Theory, J. Kral et al. (eds.), Plenum, New York (1988), 153-159. Zbl 0685.35023 MR 0986290

23. LAY, S. R. Convex Sets and Their Applications. Wiley, New York (1982). Zbl 0492.52001 MR 0655598

24. Lewis, J. L. Capacitary functions in convex rings. Arch. Ration. Mech. Anal. 66 (1977), 201-224. Zbl 0393.46028 MR 0477094

25. Longinetti, M., \& Salani, P. On the Hessian matrix and Minkowski addition of quasi-convex functions. J. Math. Pures Appl. 88 (2007), 276-292. Zbl 1144.26017 MR 2355460

26. LU, G., \& WANG, P. A uniqueness theorem for degenerate elliptic equations. In: Conference on Geometric Methods in PDE's on the Occasion of the 65th Birthday of Ermanno Lanconelli (Bologna, 2008), G. Citti et al. (eds.), Lecture Notes of Seminario Interdisciplinare di Matematica 7, Dipartimento di Matematica e Informatica, Univ. degli Studi della Basilicata, Potenza (2008), 207-222.

27. Oberman, A. M. The convex envelope is the solution of a nonlinear obstacle problem. Proc. Amer. Math. Soc. 135 (2007), 1689-1694. Zbl pre05135844 MR 2286077

28. Rockafellar, R. T. Convex Analysis. Princeton Univ. Press, Princeton, NJ (1970). Zbl 0193.18401 MR 0274683

29. SAKAGUCHI, S. Concavity properties of solutions to some degenerate quasilinear elliptic Dirichlet problems. Ann. Scuola Norm. Sup. Pisa Cl. Sci. (4) 14 (1987), 403-421. Zbl 0665.35025 MR 0951227

30. SAlAni, P. Starshapedness of level sets of solutions to elliptic PDEs. Appl. Anal. 84 (2005), 1185-1197. Zbl 1130.35055 MR 2178766

31. TOlKsdorf, P. Regularity for a more general class of quasilinear elliptic equations. J. Differential Equations 51 (1984), 126-150. Zbl 0488.35017 MR 0727034 